Kirsten Nazarkiewicz / Gesa Krämer (Hrsg.): Handbuch Interkulturelles Coaching. Konzepte, Methoden, Kompetenzen kulturreflexiver Begleitung. Mit 39 Abbildungen und 24 Tabellen. Vandenhoeck \& Ruprecht: Göttingen 2012, 415 S.

Kirsten Nazarkiewicz und Gesa Krämer unternehmen den Versuch, ein lange überfälliges Handbuch zum Interkulturellen Coaching zu schreiben, ein Konzept, das sich in den letzten Jahren einer immer größeren Beliebtheit erfreut. Dabei ist zwischen Coaching im Allgemeinen und Interkulturellem Coaching deutlich zu unterscheiden, eine Unterscheidung, die die beiden Autorinnen aufnehmen und im Kapitel 2.5 versuchen zu explizieren. Darauf wird zurück zu kommen sein.

\title{
Die Autorinnen
}

Zunächst einmal sei erwähnt, das die Autorinnen aus der Interkulturellen Coachingpraxis selbst kommen. Kirsten Nazarkiewicz (in der dritten Generation Immigrantin aus Polen, wie man aus dem Buch erfährt), leitet mit ihrer Kollegin Gesa Kämer in Frankfurt am Main das Institut Consilia (Create Culture together) und ist selbst Trainerin, Sozialwissenschaftlerin (auf dem Cover wird bedauerlicherweise nur von „Wissenschaftlerin“ gesprochen) und Coach, währen Gesa Krämer Trainerin, Coach und Körperpsychotherapeutin ist.

\section{Der Aufbau}

Zunächst einmal besticht das Werk durch einen klaren und stringenten Aufbau. Das Werk ist mit Einleitung und Schluss in insgesamt fünf Kapitel aufgeteilt: 2) Coaching kulturell - interkulturell, 3) Kompetenzfelder für kulturreflexiv arbeitende Coachs, 4) Kulturreflexive Vorgehensweisen im Coaching, 5) Schluss: Interkulturalität und Professionalität. Am Ende der einzelnen Kapitel werden Bibliographiehinweise bzw. Leseempfehlungen gegeben. Zudem werden die theoretischen Ausführungen immer wieder durch Fallbeispiele ergänzt. Angeschlossen wird das Werk durch eine sehr breite Literaturliste (S. 396ff.)

\section{Inhaltliche Bemerkungen}

Nach der Einleitung, die den Begriff thematisiert und die Problematik des Begriffs Interkulturelles Coaching aufzeigt (im Hinblick auf das virulente Problem einer "unklaren Begriffsbestimmung", einer unklaren Reichweite des Begriffs und der Frage, warum 
Coaching boomt: im Jahre 2011 nimmt Coaching in Deutschland den 1. Platz unter den Trainingsmethoden ein) geben Nazarkiewicz/Krämer auch gleich ihre Definition des Coachingbegriffs (die im Übrigen im Buch selber noch mal wiederholt wird).

Coaching ist die Antwort auf eine dynamische, sich schnell verändernde, komplexe und dadurch unübersichtlich gewordene globalisierte Arbeits- und Lebenswelt, in der es zur individuellen Orientierung der prozesshaften Einzelbegleitung bedarf. Coaching ist eine zielorientierte, systematische und zeitlich begrenzte Unterstützung eine s Entwicklungsund Veränderungsprozesses im Rahmen beruflicher oder privater Neuorientierung in Gesellschaften, die zunehmen transkulturell verfasst sind. Übergeordnetes Ziel ist die methodisch geförderte Verbesserung oder Wiederherstellung von professioneller Leistungsfähigkeit und privater Zufriedenheit des Coachingpartners mit dem Gefühl der autonomen Handlungsteuerung und des individuellen Wohlbefindens. (9)

Es scheint als hätten die Autorinnen insgesamt in erster Linie Wert auf Vollständigkeit und danach auf Prägnanz und Griffigkeit des Begriffs gelegt (eine Bemerkung, die sich durch für ein wissenschaftliches Oeuvre relativ viele Verbalisierung, also „Umschreibungen“ innerhalb des Werks selber immer wieder neu erhärten lässt). Das verweist nicht zuletzt auf den wissenschaftlichen Standort der Autorinnen zwischen Theorie und Praxis, zwischen Wissenschaft und wirtschaftlicher Anwendung (es wird nicht umsonst in dem Werk an einigen Stellen von „Kunden“ gesprochen).

Die Autorinnen gestehen die mangelnde begriffliche Erfassung des Phänomens zum Teil selbst ein, wo sie etwa schreiben, dass man in „Ermangelung einer systematischen Erforschung und theoretischen Konzeptualisierung auf die gebündelten Erfahrungen von publizierenden Kollegen angewiesen ist.“ (S.12)

Im 2. Kapitel wird versucht zum einen bestimmte Zielgruppen interkulturellem Coachings hervor zu heben wie Expatriates/Impatriates, Migranten oder ausländische Studierende. Es wird auch gleich die Frage aufgeworfen, wann Coaching interkulturell ist, die Antwort folgt dann aber erst später im Kapitel 2.5. Zudem werden Begriffe wie diversitykompetentes Coaching oder Zielgruppenansatz thematisiert.

Im Kapitel 2.2 folgt der Versuch der Darstellung des Coachingverständnisses in verschiedenen Kulturen (u.a. USA, Russland, China, Indien, Japan, etc.), das durch Fragebögen mit Fragen an Coachs in bestimmten Ländern abgestützt wird (durch Fragen etwa: „Wie wird Coaching in ihrer Kultur definiert oder verstanden?“ o.ä.). Die Autorinnen konzedieren dabei, dass es einem kulturvergleichenden Blick auf das Coaching nach wie vor an Systematik mangelt. Auffallend ist, dass hier keine verlässlichen Zahlen vom Coachingbedarf innerhalb der Kulturen selbst gebracht werden, wie populär das Coachingkonzept, abgesehen von den USA, eigentlich in den einzelnen Ländern ist. Die Verfasserinnen scheinen davon auszugehen, dass Coaching überall ähnliche Bedeutung hat oder Bedeutung haben sollte und versuchen nicht im Sinne etwa des Anti-BiasAnsatzes ihren eigenen Ausgangspunkt zunächst einmal kritisch zu reflektieren oder zu hinterfragen. Dem steht entgegen und dass sei hier positiv herausgehoben, dass die Autorinnen versuchen aufzuzeigen, welche implizierten Hypothesen die Coachs in den einzelnen Länden arbeiten (und dass dabei natürlich immer die Gefahr eines Kulturalismus besteht). (S. 50ff.). 
Der zweite, wie ich finde, wichtige Punkt, ist die Abgrenzung des Coachings von andern Formen des Trainings (ab S. 55ff.), etwa bei der Beratung ("Coaching ist egalitärer", S. 55), Mentoring („Coaching ist unabhängig“, S. 55), Einzeltraining ("Coaching ist mehr", S. 56), Supervision ("Coaching ist zielorientierter“, S. 56f.), oder Therapie (trotz gemeinsamer Methoden ist "Coaching keine Therapie“, S. 57f.)

Problematisch erscheint mir strukturell, dass die hier die eher intrakulturelle Abgrenzung der einzelnen Arten und Formate, eingeschlossen wird von Überlegungen zum interkulturellen Coaching bzw. seinen Definitionen. Der Begriff „Interkulturelles Coaching", der in Deutschland erst seit den 90er Jahren kursiert, scheint weiterhin nicht präzise gefasst zu sein, worauf die Autorinnen selbst hinweisen. Sie wählen deshalb die Lösung, die verschiedenen Ansätze interkulturellen Coachings in chronologischer Reihenfolge darzustellen, als da wären: Interkulturelles Coaching bei Konfliktmoderation und -mediation, Interkulturelles Coaching als Störungsbewältigung, Interkulturelles Coaching bei interkultureller Kommunikation, Interkulturelles Coaching als körperorientierte Persönlichkeitsentwicklung, Interkulturelles Coaching als Synergieerzeugung und Integrales Coaching (S. 60ff.)

Zudem werden von den Autorinnen drei Arten des Coachings verfolgt, die im Weiteren dann konkrete Anwendung finden: Zentral werden dabei insbesondere Formate des „kulturreflexiven Coachings“ (ein Begriff, den die Verfasserinnen selbst einführen), darunter werden Formate wie Coaching als interkulturelles Lernen, als Coaching im multikulturellen Kontext und transkulturelles Coaching subsumiert. Dabei werden tabellarisch Differenzen von trans- und interkulturell aufgelistet (S. 70). Unter Zugrundelegung unterschiedlicher Kulturbegriffe (über die sich jeder Coach nach Auffassung der Autorinnen Rechenschaft ablegen sollte) sowie des Kompetenz- und des Identitätsbegriffs werden zum Abschluss des 2. Kapitels statt Leseempfehlungen Reflexionshilfen für Coachs gegeben (S. 82)

Das 3. Kapitel verfolgt die Kompetenzfelder für kulturreflexiv arbeitende Coachs, in dem zum einen praktische Hinweise in Form eines „Cultural Backpack“ gegeben werden (S. 89) oder aber theoretische, meist bekannte Modelle wir das Kohäsionsmodell von Stefanie Rathje o.ä. vorgestellt wird. Wichtig erscheint dabei auch der performative Akt interkultureller Kommunikation unter starker Berücksichtigung des Kommunikationsaktes von den Autorinnen „creative culture together" gebannt, ein Verständniszirkel, angelehnt an den hermeneutischen Zirkel, was aber nicht weiter expliziert wird, und dem Zugrundelegung hybrider Identitäten. Etwas Ungewöhnlich erscheint in diesem Zusammenhang das Kapitel 3.1.4 Der Körper als kulturelle Konstruktion und universale Basis oder Der Körper als transkulturelles Bezugs- und Beziehungssystem, was wahrscheinlich dem Arbeitsbereich einer der Autorinnen geschuldet ist. Innerhalb des Kapitels 3.2 Methodische Kompetenzen für die Durchführung kulturreflexiver Coachings werden bekannte Methoden und Formate, darunter auch das Kulturstandardmodell vorgestellt (auch das Riemann-Thomas-Modell) sowie Kulturschockphänomenmodelle, auch diese Ausgangslage scheint kulturbedingt.

Der 4. Teil bringt zum Teil nichts viel Neues, sondern eher bereits Bekanntes, was ein Handbuch natürlich auch leisten muss. Problematisch erscheint der unkritisch benutzte 
Heimatbegriff (Heimat herstellen (179), ein Leitfaden), von dem wir ja wissen, dass er auf diese Weise sein semantisches Potential nur in der deutschen Sprache entwickelt und in anderen Sprachen (z.B. im Polnischen) keine direkte Entsprechung hat. Nicht ganz unproblematisch erscheint zudem die Übertragung von westlichen oder deutschen Modellen bzw. Formaten wie etwa Hellingers Aufstellungsarbeit (S. 231), NLP, Wahrnehmungsübungen oder das Thompson-Riemann-Modell, die vorwiegend innerhalb des intrakulturellen Coachings benutzt werden auf den interkulturellen Bereich. Hier hätte man sich weitere Differenzierungen gewünscht.

Von besonderer Bedeutung erscheint das Diversity-Rad, (S. 246) sowie das Selbst-, Differenz-, Integrationsmanagement und das 4. Kapitel Kulturreflexive Vorgehensweisen im Coaching, worin auch der Sprache breiter Raum gewidmet ist (s. 324ff.). Dass auch dieses Konzept selbst kulturspezifisch ist, ließe sich noch stärker hinterfragen, insgesamt werden meiner Meinung nach - und hier wiederhole ich mich, weil es auffallend ist - zu selten die eigenen Maßstäbe befragt, auch wenn zugestanden wird, dass sich in der Zukunft des Coachings der Fokus von Coachee auf den Coach und seine Kompetenzen verlagern wird. (S. 394).

\section{Fazit}

Trotz dieser offenkundigen Schwächen haben Nazarkiewicz und Krämer ein wichtiges Werk geschrieben, das angehenden Coachs viel wichtige Informationen liefern kann. Gerade allerdings der kulturreflexive Teil ist noch theoretisch ausbaufähig. (Anti-BiasAnsätze kommen kaum zur Sprache oder Ansätze der Kultursemiotik).

Das ist sowohl die Stärke wie die Schwäche des Buchs, zum einen wissen die Autorinnen, wovon sie reden, zum andern hätte man sich an manchen Stellen mehr theoretische Hintergründe gewünscht. Das Coaching-Konzept leidet ja in der allgemeiner Wahrnehmung ehe unter einem Theoriedefizit als unter dem Vorwurf der Unpraktikabilität oder Operationalität und gerade einem Handbuch hätte man doch sehr einen breiteren theoretischen Forschungsstand gewünscht, was auch im Schlussresümee (Kapitel 5: Interkulturalität und Professionalität nur sehr bedingt geschieht, S. 394ff.), wo die Autorinnen schreiben:

Interkulturelles Coaching ist nicht nur ein Spezialfall des Coaching, sondern kulturelle Spezifika und (inter) kulturelle Aspekte stellen eine grundlegende, vom Coach zu berücksichtigende Dimension jedes Coachingsprozesses dar. Unser Überlegungen führen zu der Schlussfolgerung, dass es hilfreich ist, Coaching als professionelles Handeln heutzutage kulturreflexiv anzugehen, um der transkulturellen Verfasstheit der internationalen Lebens- und Arbeitswelt gerecht zu werden und die in dieser Welt arbeitenden lebenden Menschen auf der Höhe der Zeit und ihrer Anforderungen zu begleiten. (S. 394)

Stephan Wolting

stephanwolting@yahoo.de

Uniwersytet im. Adama Mickiewicza w Poznaniu

DOI: $10.14746 / g 1.2015 .42 .2 .14$ 